\title{
SentiMental: An emotional profiling algorithm for identifying affect patterns in text
}

\author{
Massimo Stella, Complex Science Consulting, Via Amilcare Foscarini 2, Lecce, Italy \\ massimo.stella@inbox.com
}

SentiMental Version 0.1

December 2, 2018

\begin{abstract}
This technical report outlines the mechanisms and potential applications of SentiMental, a suite of natural language processing algorithm designed and implemented by Massimo Stella, Complex Science Consulting. The following technical report briefly outlines the novel approach of SentiMental in performing sentiment and emotional analysis by directly harnessing the whole structure of the mental lexicon rather than by using affect norms. Furthermore, this technical report outlines the direct emotional profiling and the visualisations currently implemented in version 0.1 of SentiMental. Features under development and current limitations are also outlined and discussed.

This technical report is not meant as a publication. The author holds full copyright and any reproduction of parts of this report must be authorised by the copyright holder. SentiMental represents a work in progress, so do not hesitate to get in touch with the author for any potential feedback.
\end{abstract}

\section{Introduction}

Language is a complex system, where several units can interact with each other in order to express either concrete or highly abstract concepts and meanings [1]. This phenomenon can happen at several levels, such as phonemes being combined in individual words, which can have one or multiple meanings, or rather words being assembled together to form a whole sentence, expressing even complicated concepts.

Decades of empirical and theoretical research in psycholinguistics have shown that the human mind absorbs and re-organises language in a highly dynamical structure possessing some of the features of a lexicon. In this so-called mental lexicon individual words are stored together with additional linguistic data such as synonyms, semantic features, phonological pronunciation, orthographic spelling and much more cognitive information $[2,3,1]$.

Hence, the mental lexicon represents the projection of language in the human mind and it represents a highly structured repository of language data that dynamically evolves over time [4]. Overwhelming evidence from psycholinguistics and network science have shown that the structure of the mental lexicon influences several processes related to language such as word acquisition [5, 6, 3], second language learning [7], cognitive development $[8,1]$ and cognitive impairments $[2,9]$.

Accessing the structure of the mental lexicon provides powerful information about the mental representation of the external reality, information that can be used not only for mapping language processes themselves but also for capturing a potential cognitive representation of how individuals perceive or are curious about a given entity [10] or rather associate and combine different elements according to their own creativity [11].

Important examples of the power of such approach are the recent investigations about language use over social media, where the analysis of the semantic content generated by users can shed light over the perception of specific topics. For instance, Stella and colleagues [12] mapped the sentiment intensity and the semantic content of the mental lexicons of both human users and social bots over Twitter during the voting event of the Catalan referendum. The authors found that social bots provided unique negative content and led to the reverberation of violent messages among specific groups of human users.

The above example opens the way to an exciting research direction where the synergy of semantic knowledge and sentiment analysis can reconstruct the mental lexicon of specific categories of users, identifying and reconstructing a given perception of the real world. In order to fulfil such an ambitious aim, innovative tools modelling the structure and affect of the mental lexicon are required.

\section{SentiMental: An outlook}

SentiMental is a suite of algorithms for natural language processing currently developed by Complex Science Consulting and exploiting the structure of the mental lexicon for extracting semantic information and affect from language data. SentiMental currently parses data English, Italian and Spanish. The current technical report briefly introduces the reader to the technology enabling SentiMental, its potential applications and limitations.

SentiMental is a lexicon based suite of algorithms assuming a hybrid approach to sentence parsing, focusing over the individual features of words (as in bag of words models [13]) but also mixing together features of different words (as in machine learning algorithms of sentence parsing [14]). This mixing is performed at the lexicon level and also at the sentence level. In other lexicon-based approaches such as VADER [13], individual words are scored by human annotators in isolation and then these scores are combined together for providing the sentiment polarity or the intensity of a given text. In other words, human annotators end up building a lexicon where every word has a numerical set of features, but these features depend only on one word itself. While this approach can prove powerful in many instances, it also explicitly neglects the structure of the mental lexicon, where concepts interact with each other and these interactions can end up heavily influencing lan- 
guage processing (e.g. in semantic priming or cohort effects [15]).

SentiMental follows a different approach and weights each word according to the structure of the whole mental lexicon, thus explicitly considering more information about the relational features of a whole set of concepts. This weighting is performed mainly for the explicit emotional profiling of text, which then combines weights of individual words according to syntactic rules, analogously to what other algorithms of sentiment analysis like VADER do [13]. This procedure gives in output an emotional profiling of a text, i.e. a collection of emotional intensities elicited by the combination of words in the text.

\section{Direct emotional profiling with SentiMental}

The emotional profiling of SentiMental relies on cognitive data gathered by the author during 2017 from a population of Italian young adults for the project Complex Forma Mentis (www.complexmentis.com) [16]. Participants signed a consent form for anonymous manipulation of the gathered data under a Creative Commons license CC-BY $4.0^{1}$. The cognitive data allowed to reconstruct semantic word-word relationships and clusters of words related to 8 different emotional traits, namely:

1. Anger, a secondary emotion arising as a reaction to negative stimuli and including states of irritation and rage

2. Disgust, a primary emotion expressing aversion towards something;

3. Fear, a primary emotion including states of anxiety and worry;

4. Trust, a positive emotion of openness;

5. Happiness, a general positive emotion including states of relaxation and joy;

6. Sadness, a primary emotion including states of sorrow and inhibition;

7. Surprise, an emotion including startle states of unexpectedness that can be positive, neutral or even negative;

8. Expectation, not an emotion, like Anticipation, but rather an upper-level state expressing confidence in the future and control over the environment and other emotions.

For more details about the mechanisms behind individual emotional traits, the interested reader can consult the original classification of basic emotions performed by Paul Ekman [17].

In SentiMental, positive and negative emotions are combined in order to also provide aggregated scores of sentiment intensity, i.e. detecting whether a given message is negative, neutral or positive. The weighting procedure attributes to each word a weight in relation to each of the above emotional traits by using a network representation and by clustering words in specific emotional communities. This methodology has mainly two advantages:

- Generality: word emotion is extracted from network data of general domain, so that additional emotional traits could be quantified starting from the same network representation of the

${ }^{1}$ Please contact the author for any further inquiries. mental lexicon. The above emotional traits were selected based on relevant previous work in the psychology of emotions. Ekman identified Anger, Disgust, Fear, Happiness, Sadness and Surprise as the six building blocks of emotional profiling, providing the basic reactions to evolutionary challenges and considered to be hardwired in the human mental lexicon [17]. After the advent of the circumplex model of affect [18], the work by Plutchik re-organised emotions in a space with polar opposites, namely Joy vs. Sadness, Anger vs. Fear, Trust vs. Distrust and Surprise vs. Anticipation. According to these choices, SentiMental is able to reflect the six basic emotions of Ekman [17], adding also Trust and Expectation in the analysis. It is important to underline that previous approaches to automatic emotional profiling reported that even a subset of the above emotions is suitable for the investigation of most emotional patterns present in novels and online social media $[19,20]$.

- Multilanguage Support: Since there is partial evidence in the relevant literature that the mental lexicon preserves similar structural features across linguistically related languages [7], then the same network representation can be used for achieving approximated representation of the considered emotional traits in multiple languages. The quality of the results will depend on the quality of the translation, which often requires a mixture of automatic and manual processing for distinguishing nuances in meaning that usually could get lost in translation. Human coding of translations might be time consuming but it also leads to more realistic results when tested with the parsing of specific sentences.

Notice that also other algorithms perform emotional profiling, the most notable and recent example being the smart algorithm developed by Mohammad and colleagues for the Semeval-2018 task 1: Affect in tweets [20] and focusing specifically on the shorter text of social media platforms. Notice, however, that differently from other approaches, SentiMental does not use machine learning or regression techniques, yet, but rather focuses over the structure of the mental lexicon. A performance comparison against other algorithms will be performed once SentiMental reaches version 1.0 and gets perfected with larger textual corpora.

It has to be underlined that also previous relevant automatic tools for emotional profiling constructed other lexicons of words-to-emotion mapping. The most important one is EmoLex, which was developed by Mohammad and Turney [19] and which used individual word annotations for mapping emotions to words. Although this is a powerful approach, it is also limited by the availability of annotations so that adding emotional traits requires gathering more annotated data. Instead, for SentiMental this mapping comes from the network structure of the mental lexicon itself and from the valence data gathered by the author. Another difference is that EmoLex focused on Joy rather than Happiness and on Anticipation rather than Expectation [19]. Although focusing on the same emotional traits would have allowed an easier comparison, unfortunately the current data available for SentiMental did not allow it. Joy is a more specific emotion compared to Happiness, since joy usually requires a high amount of arousal or excitation. The data currently available for the development of SentiMental did not allow to identify only concepts related 
to Joy, so a more general emotional trait including also low arousal concepts, like Happiness, was chosen, instead. An analogous issue, due to the the limited amount of data available for SentiMental, was found also for the identification of Anticipation, so that a more general trait, including also patterns of other emotions, like Expectation, was chosen, instead.

\section{Strengths of SentiMental}

It is important to underline that SentiMental does not count how many words evoke a given feeling but it rather tests the emotional relevance of each and every individual word in the mental lexicon. Even in its preliminary version, SentiMental already has the following strengths:

1. Native support to multiple languages without the need for external automatic language translations. Currently supported languages are English, Italian and Spanish.

2. Rather than performing only sentiment polarity (i.e. identifying negative or positive text), SentiMental quantifies a whole spectrum of emotional profiles for a given text, highlighting either negative emotions like anger, sadness, disgust and fear or positive emotions like happiness and trust or other emotions and emotional traits like surprise and expectation, respectively.

3. Statistical testing and filtering are performed in order to get rid of noise and even attribute statistical measures of relevance to the detected emotional patterns.

4. Sensory profiling identifies what kinds of senses are evoked by a given text. This profiling is based on data built by Complex Science Consulting.

5. Support to emoticons, which represent a rich semantic source of information for social media data and emails.

6. Support to word meaning modifiers, punctuation and syntactic rules. Syntax can greatly alter the meaning of a sentence and it represents a daunting task for naive bag of words model [13]. SentiMental processes also the syntactic and punctuation structure of sentences and updates its emotional profiling accordingly.

7. Support to word stemming in multiple languages, so that a larger number of words is recognised by SentiMental independently on different prefixes or suffixes.

\section{Features under development}

The following features of SentiMental are only partially functioning and still under development:

- Indirect emotional profiling is performed in synergy with direct emotional profiling. Indirect profiling identifies the emotions of a given textual information by considering language features such as valence, arousal and dominance. The dataset enabling indirect emotional profiling was built by Complex Science Consulting and it currently supports only Italian and English. Notice that indirect emotional profiling works in a different way compared to other smart tools visualising cognitive data such as TextSight by Hills https : //warwick.ac.uk/fac/sci/psych/ people/thills/thills/textsight/, in that the aim of the indirect emotional profiling is to extract emotions from text rather than visualising the specific dynamics of affect in text. Hence, SentiMental represents a coarse-grained alternative to more refined approaches.

- Support to spell checking and automatic correction of spelling errors, currently working only for English.

\section{Limitations of SentiMental}

The current implementation of SentiMental has the following limitations:

1. No support to gender identification. Although a growing amount of interest is devoted to understanding the gender of users in social media and their main personality traits, current logistic regression approaches suffer from their dependence over specific topics. For instance, users might be classified as men if they talk about beards and grooming, whereas the classification could prove almost equivalent to a random choice in case the users were talking about another neutral topic. Additional research about structural differences among the mental lexicaons of genders is required before robust implementations of gender profiling are brought to SentiMental.

2. Only thousands of words are parsed. The lexicons for English and Italian include 3600 stemmed words whereas the lexicon of an adult native speaker is supposed to include at least one order of magnitude more of words $[1,11]$. This limitation can be reduced by expanding the currently available lexicons with additional data.

3. No detection of humour and sarcasm. Both these elements emerge as rather complex combinations of words beyond their literal meaning [17]. It is only recently that some investigation about humour norms in English has unveiled some of the cognitive mechanisms behind humour but more data and further research is still necessary.

4. Based on young adults. SentiMental reflects the mental lexicon of younger adults and might therefore be unsuitable for modelling older people, in absence of additional data. The presence of additional data for indirect emotional profiling might solve this limitation in future versions of SentiMental.

\section{Applications of SentiMental}

\subsection{Direct emotional profiling of indi- vidual sentences}

Figure 1 reports the results of the direct emotional profiling as performed with SentiMental and relative to 5 different English sentences. Strongly negative emotions are highlighted in red. Strongly positive emotions are highlighted in blue. Ambivalent emotions and emotional traits are highlighted in grey. The bar chart of the emotional intensity for each emotional trait is what I refer to as emotional profiling 

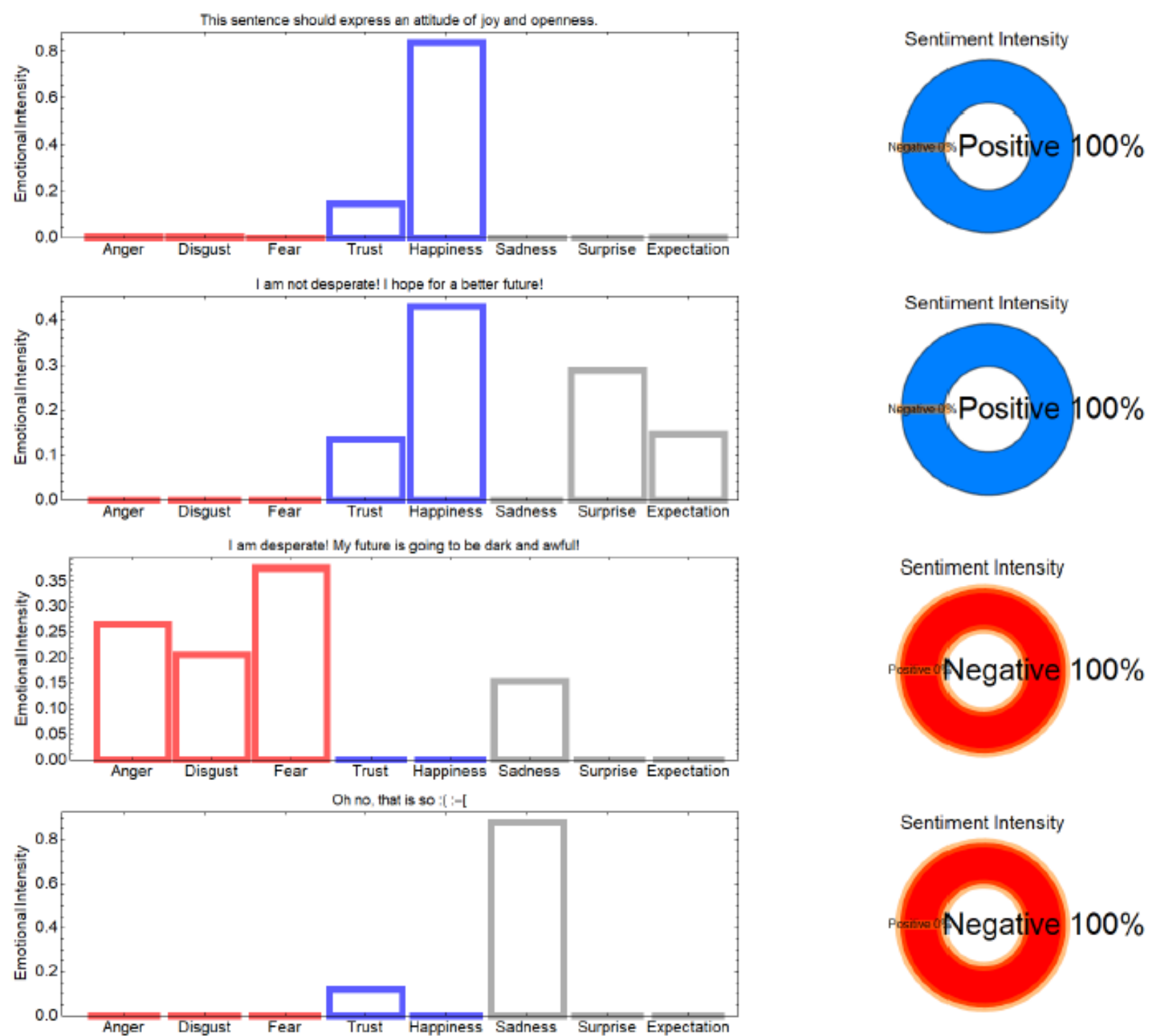

His worst fear is losing all his beloved ones but luckily, he is plenty of good friends who will never abandon him :) :)
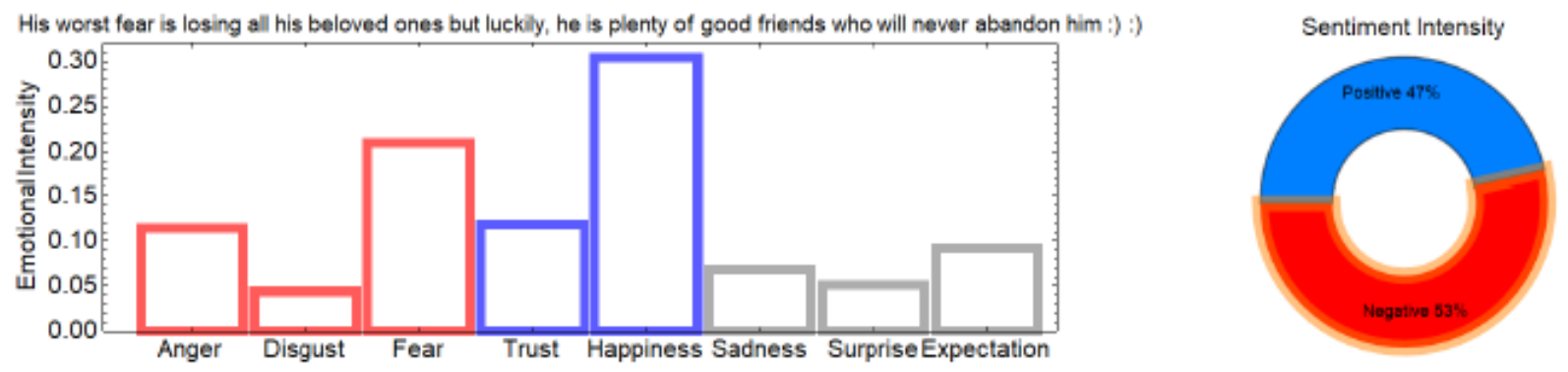

Figure 1: Direct emotional profiling of English sentences as performed with Sentimental. Different emotional states can co-exist within the same sentence, so that although two sentences can have the same sentiment intensities (reported as pie-charts), they can also differ in their emotional profiling. Furthermore, opposing emotional traits can co-occur in the same sentence, as reported in the bottom panel. 
[20]: A quantification of the strength of several emotional traits as elicited by a given sentence. Notice that this definition allows for different emotions to co-exist together in the same sentence, as it happens in the bottom panel of Figure 1 with the sentence His worse fear is losing all his beloved ones but luckily he is plenty of good friends who will never abandon him :) :). The first part of the sentence is evidently negative and evokes the emotions of fear and loneliness, whereas the second part of the sentence is positive and elicits feelings such as friendship and happiness. This dichotomy is reflected also in the sentiment polarity (reported as pie-chart plots in Figure 1). Hence, emotional profiling uncovers more emotional complexity in text than simple sentiment polarity.

Additional evidence of the richer phenomenology displayed by emotional profiling is represented by the third and fourth sentences in Figure 1. Although "I am desperate! My future is going to be dark and awful" and "Oh no, that is so:( :- " both display evident negative sentiment, the first sentence elicits mainly feelings of loneliness, desperation and fear while the second sentence sounds plainly sad. Notice also that SentiMental reports the emotion of sadness by parsing an emoticon, since Emojis also contain evident semantic content [21].

The above examples underline the higher resolution power of emotional profiling compared to standard sentiment analysis in unravelling the semantic content and the perception of text.

\subsection{Emotional profiling of textbooks}

In the digital humanities, a great deal of interest is being currently devoted to the automatic investigation of large volumes of textual corpora, a task that could be performed with SentiMental [22]. In fact, SentiMental currently works in batch mode, so that it accepts in input a given text and it outputs visualisations and data about the emotional profile of the text. The batch mode is convenient for the analysis of longer texts such as books or extensive web pages.

As a toy example for testing the power of SentiMental, the sentimental profile of the first Harry Potter book (Harry Potter and the Philosopher's Stone by J.K. Rowling) was analysed. Figure 2 highlights the emotional profiling of Fear, Happiness and Anger in the book. Emotional intensities over sentences are filtered in order to reduce noise. The lines refer to the median emotional intensities. In order to allow for a visual statistical estimation of neutrality ranges for each and every emotional trait, interquartile ranges are computed for the aggregated distribution of emotional intensities. Spikes or emotional profiles falling beyond these interquartile ranges can considered outliers according to the chosen significance level. As a baseline, we consider outliers repeated spikes of emotional intensities falling out of the interquartile range, spikes which represent strong emotions as reported in the narration.

These spikes are present in the whole narration. Interestingly, emotional spikes of happiness, anger and fear match the overall series of events narrated in the book. For instance, in the introduction, when the Dursleys are introduced by means of funny descriptions and satirical sentences, the prevalent emotion is happiness. However, at later stages when the Dursleys negate Harry's nature as a wizard, spikes of anger are registered by SentiMental. On the other hand, fear is the main emotional trait when Harry faces the dangers of Hogwarts in the Forbidden Forest later in the book.
Figure 2 agrees with a recent work by Reagan et al. [22], who investigated the overall sentiment fluctuations in novels and books. The authors found that in novels like Harry Potter and the Deathly Hallows by J.K. Rowling, the happy ending is preceded by a critical set of scenes full of dramatic and negative sentiment. This is in line with important results about the overall structure of novels in terms of climactic storytelling, where overcoming tension and fear can lead to a more meaningful happy ending [22]. SentiMental captures the same patterns here in Harry Potter and the Philosopher's Stone: The final confrontation sees the predominance of Fear and Anger, which later vanish and leave their way to Happiness in the ending of the novel.

A similar story path is reported by SentiMental in I Promessi Sposi, one of the most famous Italian novels, written by Alessandro Manzoni. The analysis of the Italian book is reported in Figure 3. The highest emotional intensities here are registered during the middle part of the novel, when one of the protagonists is involved in a popular riot in Milano (a true historical event). The happy ending of the novel is reflected by the high intensities of happiness.

These results provide evidence that the emotional profiling captured by SentiMental and reproducing the mental lexicon of the narrator is capable of capturing important fluctuations in the affect of stories, novels and books, opening the way to many further applications of SentiMental beyond social media analysis.

\subsection{Quantification of sense words}

The quantification of sensory spheres in SentiMental was specifically developed for the English language, which features sense words, i.e. words specifically evoking meanings from a certain sense. Quantifying sense words can be relevant for applications identifying different ways of providing semantic content.

Figure 4 reports an example reporting the sensory profiling of a sentence and highlighting how much, on average, the meaning of words in such sentence evoke a given sense.

\subsection{Experimenting with indirect emo- tional profiling}

A feature currently under development in SentiMental is indirect emotional profiling, where rather than directly extracting emotions from text, other features are captured instead, like valence or arousal. These features are later related to emotions by using a given psycholinguistic model of emotional states [18, 23, 17].

In here we get inspiration from the bi-dimensional model of affect developed by Posner, Russell and Peterson [24] for the representation of emotional traits in terms of valence and arousal. Valence is a feature of individual concepts expressing how positively or negatively concepts are perceived by humans. Arousal represents the level of excitation induced by a given concept. Combinations of valence and arousal can therefore describe different levels of positive/negative and excited/inhibited attitude towards the external world. Although the emotional circumplex might be an oversimplification of mood change in some cases (where psychological networks should be used instead [18]), in other cases it can represent a convenient, schematic way of reproducing basic emotional patterns [24].

SentiMental reconstructs valence and arousal by automatic estimation, based again on the mental lex- 
SentiMental Algorithm for Emotional Profiling by Massimo Stella, Complex Science Consulting

Harry Potter and the Philosopher's Stone by J.K. Rowling Flow of Events

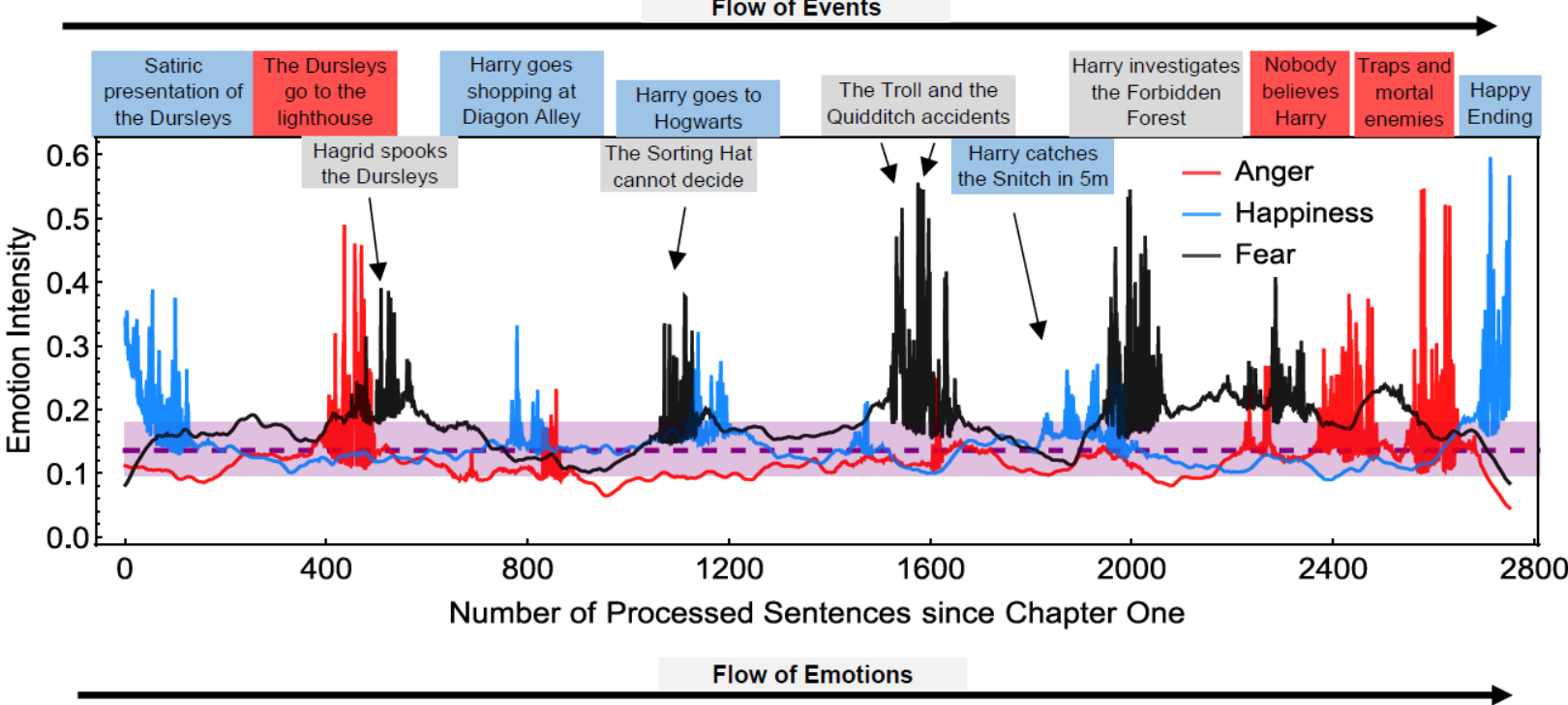

Figure 2: Direct emotional profiling of the flow of narration for the first Harry Potter book by J.K. Rowling in English. The emotional intensities, as extracted from the mental lexicon of Sentimental, are reported over the series of events narrated in the book. The plot focuses on the emotional traits of Anger, Happiness and Fear. The top plot reports the flow of events.

SentiMental Algorithm for Emotional Profiling by Massimo Stella, Complex Science Consulting

I Promessi Sposi by Alessandro Manzoni Flow of Events

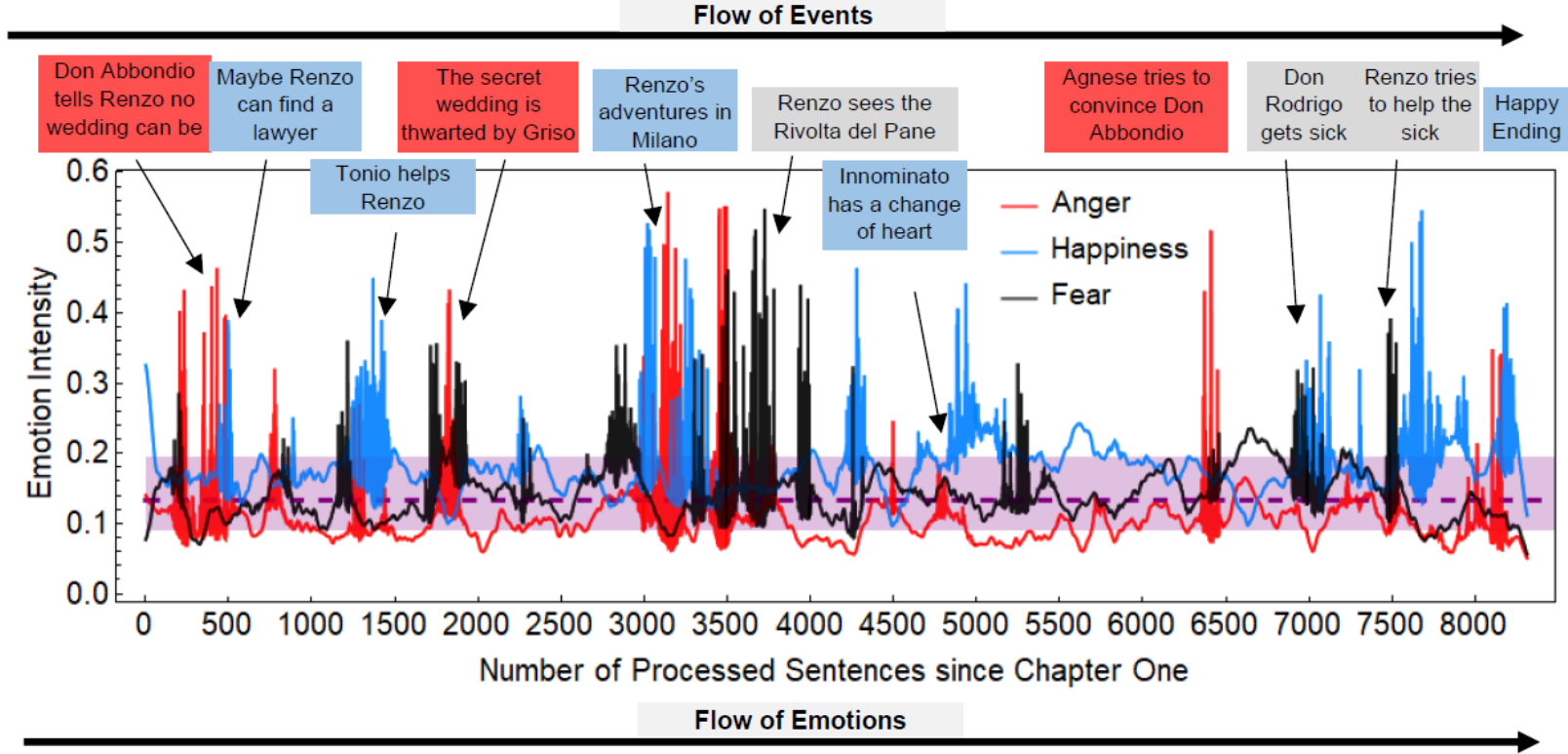

Figure 3: Direct emotional profiling of the flow of narration for I Promessi Sposi by Alessandro Manzoni. The emotional intensities, as extracted from the mental lexicon of SentiMental, are reported over the series of events narrated in the book. The plot focuses on the emotional traits of Anger, Happiness and Fear. The top plot reports the flow of events.

icon structure. However, in comparison with the original circumplex representation, SentiMental's visualisation of emotional patterns transforms a sentence into a visually distributed representation of emotions, featuring statistical testing against neutrality bounds and also reporting valence and arousal intensities. Some examples are reported in Figure 3. The sentence on the left features negative, inhibitory concepts and hence highlights emotions of boredom and hatred. The sentence on the right features a mix of alarmism (related to the desire of fighting) but also positive concepts of serene determination that would otherwise be difficult to spot in a visualisation based only on median or mean values at the level of the whole sentence. In fact, the visualisation adopted for SentiMental differs from the one of other tools relying on the circumplex of affect, such as TweetWiz by Healey and Ramaswamy https://www.csc2.ncsu. edu/faculty/healey/tweet_viz/, in that rather than considering average values, the whole profiles of valence and arousal from a given sentence are reported and tested, instead.

Another visualisation is currently under development in SentiMental, performing indirect emotional 

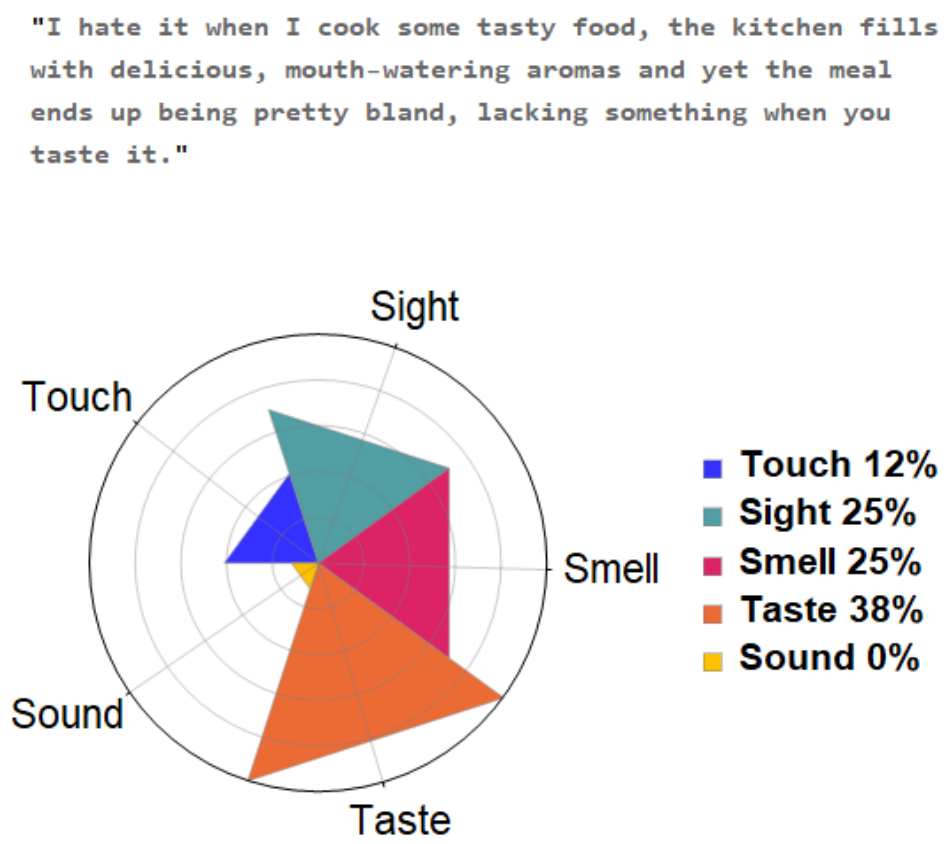

Figure 4: Sensory profiling of a sentence as performed by SentiMental. The percentages represent how much the sentence elicited a given sentence. The visualisation uses a radar plots for presenting the results in sensory space.
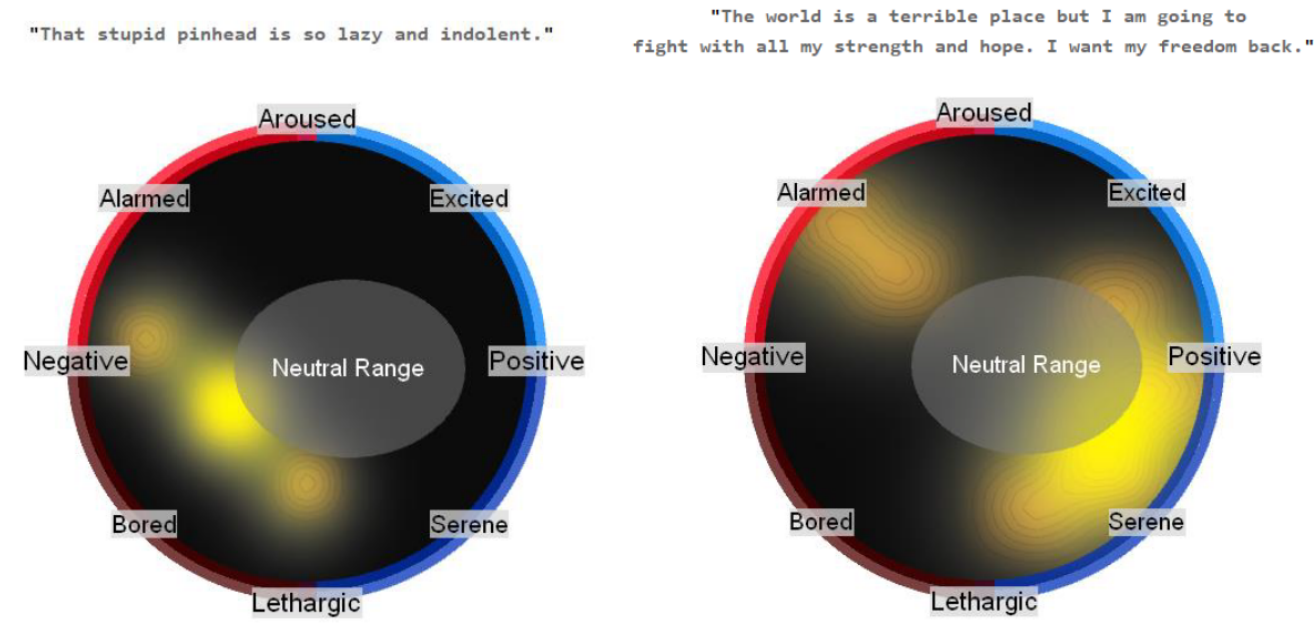

Figure 5: Indirect emotional profiling as performed by SentiMental. Individual features of words are transformed into a bidimensional profile in a space where individual directions report individual emotions. On the $\mathrm{x}$-axis, the positive or negative perception of words is reported, whereas the $\mathrm{y}$-axis features the general level of excitement. Yellow marks reports peaks of perception/excitement. The neutral range reported here is obtained by considering the whole distribution of all words in the mental lexicon represented in SentiMental. Yellow marks falling outside of the neutral range represent outliers and indicate emotional patterns according to their position in the plot. Black indicates a lack of emotional intensity. Yellow indicates a strong emotional intensity.

profiling in a larger emotional space [24]. Although this representation adopts innovative tools for the investigation of psycholinguistic patterns, it also requires further validation and empirical testing, hence the need for further development and the choice of not reporting it on here yet.

\section{Copyright Disclaim}

In agreement with the Open Science Foundation policies for licensing pre-prints and with the CC-BY 4.0 license of the data (see www.complexmentis. com) behind SentiMental, this technical report is released with no license, indicating that the author holds full copyright (see also http://help.osf.io/ $\mathrm{m} /$ preprints/l/726873-preprint-faqs).

Furthermore, no adaption or redistribution of the contents of this technical report can be performed without explicit permission of the author.

SentiMental is an algorithm designed and implemented by Massimo Stella as one of the flagship projects of Complex Science Consulting, Via Amilcare Foscarini 2, Lecce, Italy.

The current technical report does not correspond to either a publication or a public release of the software, which is still under development. However this technical report represents evidence of the design and potential applications of the SentiMental software in different contexts, so that its publication on the OSF repositories can hopefully promote constructive discussion and technological advancement.

For any further inquiries or if interested in scientific consulting as performed by Complex Science Consulting, please get in touch with the author. 


\section{Acknowledgements}

The author acknowledges Dr. Siew and Prof. Hills for their feedback on the analysis of the Harry Potter novel and TextSight.

\section{References}

[1] Cynthia SQ Siew, Dirk U Wulff, Nicole Beckage, and Yoed Kenett. Cognitive network science: A review of research on cognition through the lens of network representations, processes, and dynamics.

[2] Michael S Vitevitch and Nichol Castro. Using network science in the language sciences and clinic. International journal of speech-language pathology, 17(1):13-25, 2015.

[3] Thomas T Hills and Cynthia SQ Siew. Filling gaps in early word learning. Nature Human Behaviour, 2(9):622, 2018.

[4] Dirk U Wulff, Thomas Hills, and Rui Mata. Structural differences in the semantic networks of younger and older adults.

[5] Massimo Stella, Nicole M Beckage, and Markus Brede. Multiplex lexical networks reveal patterns in early word acquisition in children. Scientific Reports, 7:46730, 2017.

[6] Massimo Stella and Manlio De Domenico. Distance entropy cartography characterises centrality in complex networks. Entropy, 20(4):268, 2018.

[7] Brent Wolter. Comparing the 11 and 12 mental lexicon: A depth of individual word knowledge model. Studies in second language acquisition, 23(1):41-69, 2001.

[8] Massimo Stella, Nicole M Beckage, Markus Brede, and Manlio De Domenico. Multiplex model of mental lexicon reveals explosive learning in humans. Scientific reports, 8(1):2259, 2018.

[9] Nichol Castro and Massimo Stella. The multiplex structure of the mental lexicon influences picture naming in people with aphasia. 2018.

[10] Perry Zurn and Danielle S Bassett. On curiosity: A fundamental aspect of personality, a practice of network growth. Personality Neuroscience, 1 .

[11] Yoed N Kenett, Orr Levy, Dror Y Kenett, H Eugene Stanley, Miriam Faust, and Shlomo Havlin. Flexibility of thought in high creative individuals represented by percolation analysis. Proceedings of the National Academy of Sciences, page 201717362, 2018.

[12] Massimo Stella, Emilio Ferrara, and Manlio De Domenico. Bots increase exposure to negative and inflammatory content in online social systems. Proceedings of the National Academy of Sciences, page 201803470, 2018.

[13] CJ Hutto Eric Gilbert. Vader: A parsimonious rule-based model for sentiment analysis of social media text. In Eighth International Conference on Weblogs and Social Media (ICWSM14). Available at (20/04/16) http://comp. social. gatech. edu/papers/icwsm14. vader. hutto. pdf, 2014.
[14] Bingwei Liu, Erik Blasch, Yu Chen, Dan Shen, and Genshe Chen. Scalable sentiment classification for big data analysis using naive bayes classifier. In Big Data, 2013 IEEE International Conference on, pages 99-104. IEEE, 2013.

[15] Massimo Stella. Cohort and rhyme priming emerge from the multiplex network structure of the mental lexicon. 2018.

[16] Catherine Cramer, Ralucca Gera, Evelyn Panagakou, Mason A Porter, Hiroki Sayama, Lori Sheetz, Massimo Stella, and Stephen Uzzo. Proceedings of netscied 2018 .

[17] Paul Ekman. Basic emotions. Handbook of cognition and emotion, pages 45-60, 1999.

[18] Randy J Larsen and Edward Diener. Promises and problems with the circumplex model of emotion. 1992.

[19] Saif M Mohammad and Peter D Turney. Emotions evoked by common words and phrases: Using mechanical turk to create an emotion lexicon. In Proceedings of the NAACL HLT 2010 workshop on computational approaches to analysis and generation of emotion in text, pages 2634. Association for Computational Linguistics, 2010.

[20] Saif Mohammad, Felipe Bravo-Marquez, Mohammad Salameh, and Svetlana Kiritchenko. Semeval-2018 task 1: Affect in tweets. In Proceedings of The 12th International Workshop on Semantic Evaluation, pages 1-17, 2018.

[21] Petra Kralj Novak, Jasmina Smailović, Borut Sluban, and Igor Mozetič. Sentiment of emojis. PloS one, 10(12):e0144296, 2015.

[22] Andrew J Reagan, Lewis Mitchell, Dilan Kiley, Christopher M Danforth, and Peter Sheridan Dodds. The emotional arcs of stories are dominated by six basic shapes. EPJ Data Science, 5(1):31, 2016.

[23] Robert Plutchik. The psychology and biology of emotion. HarperCollins College Publishers, 1994.

[24] Jonathan Posner, James A Russell, and Bradley S Peterson. The circumplex model of affect: An integrative approach to affective neuroscience, cognitive development, and psychopathology. Development and psychopathology, 17(3):715-734, 2005. 